\title{
PREVELANCE OF ANTI-TPO ANTIBODY IN TYPE-1 DIABETES AND THYROID DYSFUNCTION IN TPO ANTIBODY POSITIVE DIABETICS.
}

\author{
Ganesan Subramanyam, Josephine Latha Pushparaj
}

1. Assistant Professor, Department of Biochemistry, K. A. P. V. Government Medical College, Tiruchirapalli, Tamilnadu, Dr. M. G. R. Medical University.

2. Assistant Professor, Department of Biochemistry, K. A. P. V. Government Medical College, Tiruchirapalli, Tamilnadu, Dr. M. G. R. Medical University.

\section{CORRESPONDING AUTHOR}

Dr. Ganesan Subramanyam,

41, V. O. C. Nagar, Angarai, Lalgudi,

Tiruchirappalli-621 703,

E-mail: drganesans@gmail.com, sganesandr@yahoo.com,

Ph: $00919943945369,00918903625415$.

ABSTRACT: BACKGROUND: The appearance of TPO-Abs precedes thyroid dysfunction and increases in autoimmune diseases like type1diabetes.Thyroid peroxidase (TPO) antibodies are one of the major secondary antibodies associated with autoimmune thyroid disease and can be used as diagnostic marker. The prevalence of thyroid auto antibodies is increased when patients have non-thyroid autoimmune diseases such as type 1 diabetes and pernicious anemia. Thyroid dysfunction is common among diabetic patients and can produce metabolic disturbances. Therefore, regularly screening diabetic patients allows early treatment. OBJECTIVE: The objective of our study is to measure TPO-Abs in young Type-1Diabeticindividuals and to find Thyroid abnormalities in TPO-Abs positive individuals. MATERIALS AND METHODS: This was a cross-sectional study conducted at a rural clinic in Tiruchirappalli, Tamil Nadu. 60 persons in the age group of 10 to 35 years were selected for this study. Fasting blood samples were collected from the study population and glucose, lipid profile, thyroid profile and TPO- Ab were estimated using standard kits by standard methods. RESULTS:16 persons showed high levels of anti TPO-Abs(> 40 IU).In the anti TPO-Ab Positive group, all values were statistically significant according to the Pearson $\mathrm{R}$ formula $\mathrm{P}<0.001$. There was significant correlation between age and anti TPO-Ab level, between weight, BMI and TPO Positive and Negative levels, as per the TTest $\mathrm{P}<0.001 .56 .30 \%$ of anti TPO-Ab Positive subjects had high TSH. CONCLUSION: Our results indicate that thyroid dysfunctionis common in Type-1 diabetes but more in anti TPO-Ab positive subjects. Hence all Type- 1 diabetic individuals should undergo annual screening of serum anti TPO-Ab and TSH measurement in anti TPO-Ab positive individuals.

KEY WORDS: Type-1 diabetes, lipid profile, thyroid profile, TSH and TPO-Ab

INTRODUCTION: There are a number of auto antibodies associated with the autoimmune thyroid diseases, which are characterized as either primary or secondary antibodies. Primary antibodies are directly pathogenic and often directed against cell membrane receptors, whilst secondary antibodies do not appear to be involved in pathogenesis but can serve as useful diagnostic markers for the presence of autoimmune thyroid disease. Thyroid peroxidase (TPO) antibodies are one of the major secondary antibodies associated with autoimmune thyroid disease.

TPO was previously known as thyroid microsomal antigen (1). It is a $107 \mathrm{KD}$ enzyme which is involved in thyroid hormone synthesis. TPO is located both on the cell surface and within the

Journal of Evolution of Medical and Dental Sciences/Volume1/Issue5/November-2012Page-668 
cytoplasm of thyroid acinar cells, bound to the vesicle which transports newly synthesized thyroglobulin, where it is involved in the iodination of thyroglobulin. High affinity antibodies (predominantly IgG) directed against TPO is found at elevated levels in the serum of patients with autoimmune thyroid disease such as Graves's disease, Hashimoto's thyroiditis, Diabetes mellitus and myxedema. Autoimmune thyroid disease (AITD) causes cellular damage and alters thyroid gland function by humoral and cell-mediated mechanisms (1).

Cellular damage occurs when sensitized T-lymphocytes and/or auto antibodies bindto thyroid cell membranes causing cell lysis and inflammatory reactions. Alterations in thyroid gland function result from the action of stimulating or blocking auto antibodies on cell membrane receptors. Three principal thyroid auto antigens are involved in AITD. These are thyroperoxidase (TPO), thyroglobulin (Tg) and the TSH receptor (1). Other auto antigens, such as the Sodium iodide Symporter have also been described, but as yet have no diagnostic role in thyroid autoimmunity.

CLINICAL SIGNIFICANCE OF THYROID AUTOANTIBODIES: TPO antibodies (TPO-Abs) appear to be involved in the tissue destructive processes associated with the hypothyroidism observed in Hashimoto's and atrophic thyroiditis(2). The appearance of TPO-Abs usually precedes the development of thyroid dysfunction. Some studies suggest that TPO-Abs may be cytotoxic to the thyroid. TPO-Ab and/or Tg-Ab are frequently present in the sera of patients with AITD.

The prevalence of thyroid auto antibodies is increased when patients have non-thyroid autoimmune diseases such as type 1diabetes and pernicious anemia. Ageing is also associated with the appearance of thyroid auto antibodies and increased prevalence of AITD.

THYROID DISEASE AND DIABETES: Thyroid disease is widespread and prevalence increases with advancing age. However, as assessing thyroid function is reliable and inexpensive, certain high risk groups such as neonates, the elderly and diabetics - should undergo regular screening.

Diabetes mellitus (DM) is a multisystem disease and is a chronic disease of carbohydrate, fat, and protein metabolism caused by the lack of insulin. In type 1 diabetes, insulin is functionally absent because of the destruction of the beta cells of the pancreas. Type 1 DM occurs most commonly in juveniles but can occur in adults, especially in those in their late 30 s and early 40s.Diabetes mellitus is thought to be, in some cases, an auto-immune disease caused when antibodies attack certain cells of the pancreas, affecting the production of insulin. In patients or families where auto-immune thyroid disease exists, this type of diabetes mellitus may develop, sometimes in younger members of the family. This should be suspected particularly if symptoms of tiredness and weight loss develop together with increased thirst and passing of large volumes of urine. Clinically, thyroid dysfunction may undermine diabetes control. For example, hyperthyroidism may worsen glycemic control and increase insulin requirements. Indeed, thyrotoxicosis may unmask subclinical diabetes. While hypothyroidism markedly alters carbohydrate metabolism, such changes are rarely clinically significant. However, as less insulin is degraded, the exogenous insulin requirement may be lower. Moreover, hypothyroidism often produces dyslipidemias, including elevated triglyceride and low- density lipoprotein (LDL) cholesterol concentrations. Therefore, hypothyroidism can exacerbate coexisting dyslipidemias in type 2 diabetes. Thyroxin reverses these lipid abnormalities. But diagnosing thyroid dysfunction can be difficult. For example, poor glycemic control produces symptoms similar to hyperthyroidism, such as weight loss despite increased appetite as well as fatigue. Clinicians need to be careful not to confuse severe diabetic 
nephropathy and hypothyroidism, both producing edema, fatigue, pallor and weight gains. Finally, poorly controlled diabetes may alter thyroid function(3).

Against this background, the serum TSH immunoassay offers the most reliable and sensitive screening test for thyroid dysfunction. However, screening for anti-thyroid peroxidase (TPO) antibodies in people with type 1 diabetes may predict autoimmune thyroid disorders.

Management is generally similar to that in the non-diabetic population. However, Lthyroxin therapy may exacerbate angina by increasing myocardial contractility and heart rate. Clinicians should consider treating subclinical hypothyroidism if patients either have elevated serum LDL cholesterol exacerbated by hypothyroidism or detectable serum anti-TPO antibodies.

Thyroid dysfunction is common among diabetic patients and can produce metabolic disturbances. Therefore, regularly screening diabetic patients allows early treatment(4). Type 1 patients expressing anti-TPO antibodies should be screened annually(5). In anti-TPO negative patients, a TSH assay every two to three years suffices. Among patients suffering from type 2 diabetes, clinicians should consider a TSH at diagnosis and then at least every five years.

\section{OBJECTIVE OF THE STUDY:}

1. To detect and measure TPO-Ab in young Type-1 DM individuals.

2. To find out the Thyroid abnormalities in TPO-Ab positive individuals in IDDM - using Thyroid function tests.

MATERIALS AND METHODS: This was a cross-sectional study conducted at a rural clinic in Tiruchirappalli, Tamil Nadu. 60 persons in the age group of 10 to 35 years were selected for this study. Subjects with any anti-thyroid treatment (or) hormone (or) lipid lowering drugs were excluded from the study. All the participants were enquired by a questionnaire about their complaints and physically examined to rule out features suggesting any hypo (or) hyper thyroid status.

Fasting (10-12 hrs of fasting) blood specimen was collected from each participant and analyzed for the following parameters. Plasma fasting glucose level was estimated by GOD/POD method in auto analyzer. Serum tri-iodothyronine, serum thyroxin and thyroid stimulating hormone were estimated by enzyme immunoassay method. Serum total cholesterol, serum triglycerides were estimated by enzymatic methods and serum high density lipoprotein cholesterol biphosphotungstate method using standard kits in auto-analyzer in the fasting serum sample. Serum low density lipoprotein cholesterol was estimated using Friedwald's formula. Estimation of TPO antibodies by accubind ELISA micro wells, a sequential ELISA method. Normal TPO-Ab level is below Forty (40) IU / ml

RESULTS: In our study 60 individuals withType-1 DM in the age group of 10 to 35 years were included. Based on the level of anti TPO antibody, the biochemical parameters T3, T4, TSH and lipid profile were tabulated, statistically analyzed and evaluated. In this study 16 persons showed high levels of anti TPO- Ab greater than 40 IU. (Table-1/ chart-1).

In anti TPO-Ab Positive Individuals, T3 levels were low in 5 persons (31.3\%) (Chart 2).T4 levels were low in 6 persons (37.5\%) (Chart 3) and TSH levels high in 9 persons (56.3\%) (Chart 4) (Table- 2).

Journal of Evolution of Medical and Dental Sciences/Volume1/Issue5/November-2012Page-670 
The value of high TSH was statistically significant according to the Pearson R formula P $<0.001$.There was no correlation between duration of IDDM and anti TPO Ab level (Table 3).There was no statistical correlations between high anti TPO Ab titer and Lipid profile (Table4).TPO Ab titer was positively correlated with TSH and negatively correlated with T3 and T4 significantly (Table-5).

Table-3 shows overall correlation between TPO, Age, Duration of the Disease, Blood Sugar level and BMI. There was no significant correlation between Blood-Sugar level and TPO level. Prevalence of TPO-Ab with $95 \% \mathrm{CI}$ is 23 to 30 . In our study that was $26.7 \%$.

DISCUSSION: Auto immune mechanisms are involved in many cases of Type-1 DM (6). The role of screening for thyroid levels in Type-1 DM is controversial. Substantial prevalence of thyroid abnormalities were noted in patients with Type-1 diabetes mellitus (7).Hypothyroidism is more common in females than males (2). TPO positive patients are more prone to develop thyroid dysfunction (8). This statement correlates with our study. Thyroperoxidase being responsible for iodination of tyrosine moieties is essential for active thyroid hormone T4 and T3 synthesis. When inhibited by anti TPO Abs, the active T4, T3 synthesis decreases, resulting in low T4, T3 level and there is a compensatory increase in TSH level, which with increased anti TPO titer and duration leads to deterioration of thyroid function from sub clinical dysfunction to fully manifest clinical hypothyroidism.

Our results indicate that all Type-1 diabetic individuals with positive TPO-Ab should undergo annual screening of serum TSH measurement to detect asymptomatic thyroid dysfunction. In Type- 1 diabetes $21.6 \%$ has high level of antibodies to TPO and TG. Thyroid autoimmunity was more common in girls and this predominance was observed in all age groups. In a study to detect sub-clinically associated AITD, $22 \%$ of patients showed thyropathy with the assessment of thyroid auto antibodies and TSH.

The screening for auto antibodies in Type- 1 diabetic patients will reveal sub-clinical cases of AITD. The sub-clinical thyroid dysfunction has no influence on diabetic control. There is a need for regular follow up of patients with positive auto antibodies to detect further deterioration of other organs(9).

Type-1 Diabetic patients with thyroid abnormalities have shown an increase in thyroid volume ultrasonographically (10). The expression of involvement of the thyroid in an auto immune disorder is not limited to the islet cells.

The use of high sensitive immunometric methods in clinical laboratories to assay anti thyroid anti bodies had expanded in recent years. The agreement of qualitative results is close to $97 \%$ for anti-TPO (11). In individuals with Type-1 DM, the measurements of anti TPO-Abs and TSH are the most efficient and cost effective combination of screening tests in the early detection of AITD (5 and 10).

The positive predictive value of anti TPO-Abs and TSH is $90 \%$. In our study there was increased level of anti TPO-Abs and increased level of TSH and low T4 and T3 level indicating sub clinical hypo thyroidism (12,13 and 17).

In a three years follow up study of Type-1 DM the prevalence of thyroid dysfunction increased from 5 to $8 \%$. The prevalence of TPO-Abs is unchanged. All Type- 1 DM patients with increased TSH level has ultra sound abnormalities(14) while ultra sound abnormalities were not always associated with increase in TSH level. Thyroid ultrasound abnormalities were a sensitive but nonspecific marker of auto immune thyroid diseases. This shows that it is unsuitable for screening purposes.

Journal of Evolution of Medical and Dental Sciences/Volume1/Issue5/November-2012Page-671 
So we recommend regular annual screening of serum TSH in the follow up of young Type-1 DM (15 and 16). Sub clinical phase of thyroid dysfunction in Type-1 diabetes due to auto antibody positivity did not affect control of diabetes while fully manifested hyper, hypothyroidism impair diabetes control. So there is no influence on diabetic control(9).In our study fasting blood glucose level in cases with positive TPO-Abs did not reveal statistical significance which is similar to previous studies.

CONCLUSION: Type-I DM being a chronic auto immune disease is associated with different auto-antibodies to thyroid, viz anti TPO-Abs, anti TG- Abs and anti-thyroid antibodies. The Anti TPO- Abs is the autoimmune parameter measured in Type-1 DM patients. In reference 16, the authors proposed the following screening protocol Thyroid auto - antibodies should be measured at diagnosis of Type-1 DM and should be repeated if TSH level exceeded the reference range and free $\mathrm{T} 4$ and $\mathrm{TSH}$ level should be measured at diagnosis and annually thereafter.

While the other studies suggest, Type-1 DM young patients with anti TPO Abs develop clinical thyroid disease with a mean latent interval of 10 years from the onset of Type- $1 \mathrm{DM}$, during which sub clinical thyroid dysfunction occurs and it should be suffice to measure TSH level annually in anti TPO positive Type-1 DM patients with which we concur.

\section{REFERENCE:}

1. Manorama Swain, Truptirekha Swain, Binoy Kumar Mohanty. Autoimmune Thyroid Disorders - An Update. Indian Journal of Clinical Biochemistry. 2005; 20 (1):9-17

2. SeyedMahmoodGhoraishian, SeyedHosseinHekmatiMoghaddam and Mohammad Afkhami. Relationship Between Anti-Thyroid Peroxidase Antibody and Thyroid Function Tests.W J Med Sci2006 Jan-Jun; 1(1) : 44-47

3. Moayeri $\mathrm{H}$ and Rabbani A.Prevalence of autoantibodies to thyroid Peroxidase and autoimmune thyroid disease in Type I diabetes mellitus.ActaMedicaIranica. 2004;42(4): 267-271.

4. Riley WJ, Maclaren NK, Lezotte DC, Spillar RP, Rosenbloom AL. Thyroid autoimmunity in insulin-dependent diabetes mellitus: the case for routine screening. J Pediatr . 1981;99:350-354.

5. Kordonouri O, Klinghammer A, Lang EB, Grüters-Kieslich A, Grabert M, Holl RW. Thyroid autoimmunity in children and adolescents with type 1 diabetes: a multicenter survey. Diabetes Care 2002Aug; 25(8):1346-1350.

6. Shimura.H, Kobayashi.T, Autoimmune Endocrine Diseases. JMAJ. 2004 Sep;47(9): 419424.

7. Guillermo E. Umpierrez, Md,Kashif A. Latif, Md,Mary Beth Murphy, Rn, Ms, Cde, Mba, Helen C. Lambeth, Rn, Frankie Stentz, Phd, Andrew Bush, Phd, Abbas E. Kitabchi, Phd, Md Thyroid Dysfunction in Patients With Type 1 Diabetes. Diabetes Care 26:1181-1185, 2003

8. Bjoro T, Holmen J, Kruger Oet al. Prevalence of thyroid disease, thyroid dysfunction and thyroid peroxidase antibodies in a large, unselected population. The Health Study of Nord-Trondelag (HUNT). Eur. J. Endocrinol. 2000; 143: 639-647.

9. Prázný M, Škrha J, Límanová Z, Vaníčková Z, HilgertováJ, Prázná J et al. Screening for Associated Autoimmunity in Type 1 Diabetes Mellitus With Respect To Diabetes Control.Physiol. Res. 2005; 54: 41-48. 
10. BianchiG, MontanariP, FabbriA, Gamberini A, Zoli M,MarchesiniG. Thyroid volume in type 1 diabetes patients without overt thyroid disease.ActaDiabetologica. 1995March; 32(1): 49-52.

11. Tozzoli R,Bizzaro N, Tonutti E, PradellaM,Manoni F,Vilalta D et al. Immunoassay of AntiThyroid Autoantibodies: Clinical Chemistry and Laboratory Medicine 2002 Jun;40(6):568-573.

12. Bilimoria KY, Pescovitz OH, DiMeglio LA. Autoimmune thyroid dysfunction in children with type 1 diabetes mellitus: screening guidelines based on a retrospective analysis. PediatrEndocrinolMetab. 2003 Oct-Nov; 16(8):1111-1117.

13. Sarah J. Glastras, Maria E. Craig, Charles F. Verge,Albert K. Chan, Janine M.Cusumano,

14. Kim C. Donaghue. The Role of Autoimmunity at Diagnosis of Type 1 Diabetes in the

15. Development of Thyroid and Celiac Disease and Microvascular Complications.Diabetes Care. 2005September; 28(9):2170-2175

16. HansenD, BennedbaekFN, Hoier-MadsenM, HegedusL, JacobsenBB. A prospective study of thyroid function, morphology and autoimmunity in young patients with type 1 diabetes. European Journal of Endocrinology. 2003; 148(2): 245-251

17. Lorini R, d'Annunzio G, Vitali L, Scaramuzza A. IDDM and autoimmune thyroid disease in the pediatric age group. J PediatrEndocrinolMetab. 1996 Mar; 9(1):89-94.

18. Kordonouri O, Hartmann R, DeissD, WilmsM,Grüters-KieslichA.Natural course of autoimmune thyroiditis in type 1 diabetes: association with gender, age, diabetes duration, and puberty. Archives of Disease in Childhood. 2005;90:411-414

19. De Graaff L.C.G. , Martín-Martorell P, Baan J, Ballieux B, Smit J.W.A., Radder J.K. Longterm follow-up of organ-specific antibodies and related organ dysfunction in type 1 diabetes mellitus. Netherlands The Journal of Medicine. 2011 Feb; 69(2) : 66-71.

\section{Chart-1}

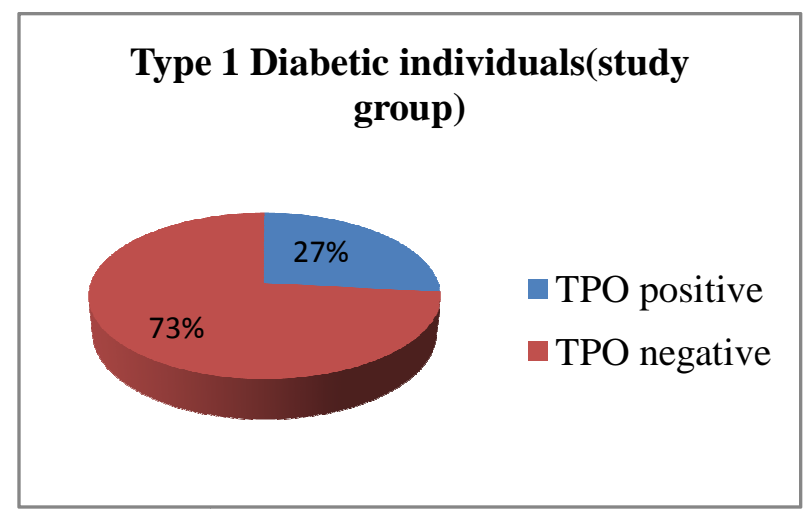

\begin{tabular}{|l|c|c|}
\hline & Frequency & Percent \\
\hline Negative & 44 & 73.3 \\
\hline Positive & 16 & 26.7 \\
\hline Total & 60 & 100.0 \\
\hline
\end{tabular}

Table 1 Percentage of TPO-AbposItive\& negative type I diabetics 
Table-2 TPO-Ab and thyroid function tests(T3, T4 and TSH)

\begin{tabular}{|c|c|c|c|c|c|c|}
\hline & \multicolumn{3}{|c|}{ TPO-Ab negative } & \multicolumn{3}{|c|}{ TPO-Ab positive } \\
\hline & Low & Normal & High & Low & Normal & High \\
\hline $\begin{array}{l}\text { T3 } \\
\text { count } \\
\text { percent }\end{array}$ & $\begin{array}{l}3 \\
6.8 \%\end{array}$ & $\begin{array}{l}41 \\
93.2 \%\end{array}$ & & $\begin{array}{l}5 \\
31.3 \%\end{array}$ & $\begin{array}{l}11 \\
68.8 \%\end{array}$ & \\
\hline $\begin{array}{l}\text { T4 } \\
\text { count } \\
\text { percent }\end{array}$ & $\begin{array}{l}3 \\
6.8 \%\end{array}$ & $\begin{array}{l}41 \\
93.2 \%\end{array}$ & & $\begin{array}{l}6 \\
37.5 \% \\
\end{array}$ & $\begin{array}{l}10 \\
62.5 \%\end{array}$ & \\
\hline $\begin{array}{l}\text { TSH } \\
\text { count } \\
\text { percent }\end{array}$ & & $\begin{array}{l}42 \\
95.5 \%\end{array}$ & $\begin{array}{l}2 \\
4.5 \%\end{array}$ & $\begin{array}{l}1 \\
6.3 \%\end{array}$ & $\begin{array}{l}6 \\
37.5 \% \\
\end{array}$ & $\begin{array}{l}9 \\
56.3 \%\end{array}$ \\
\hline
\end{tabular}

\section{Chart-2}

\section{Chart-3}
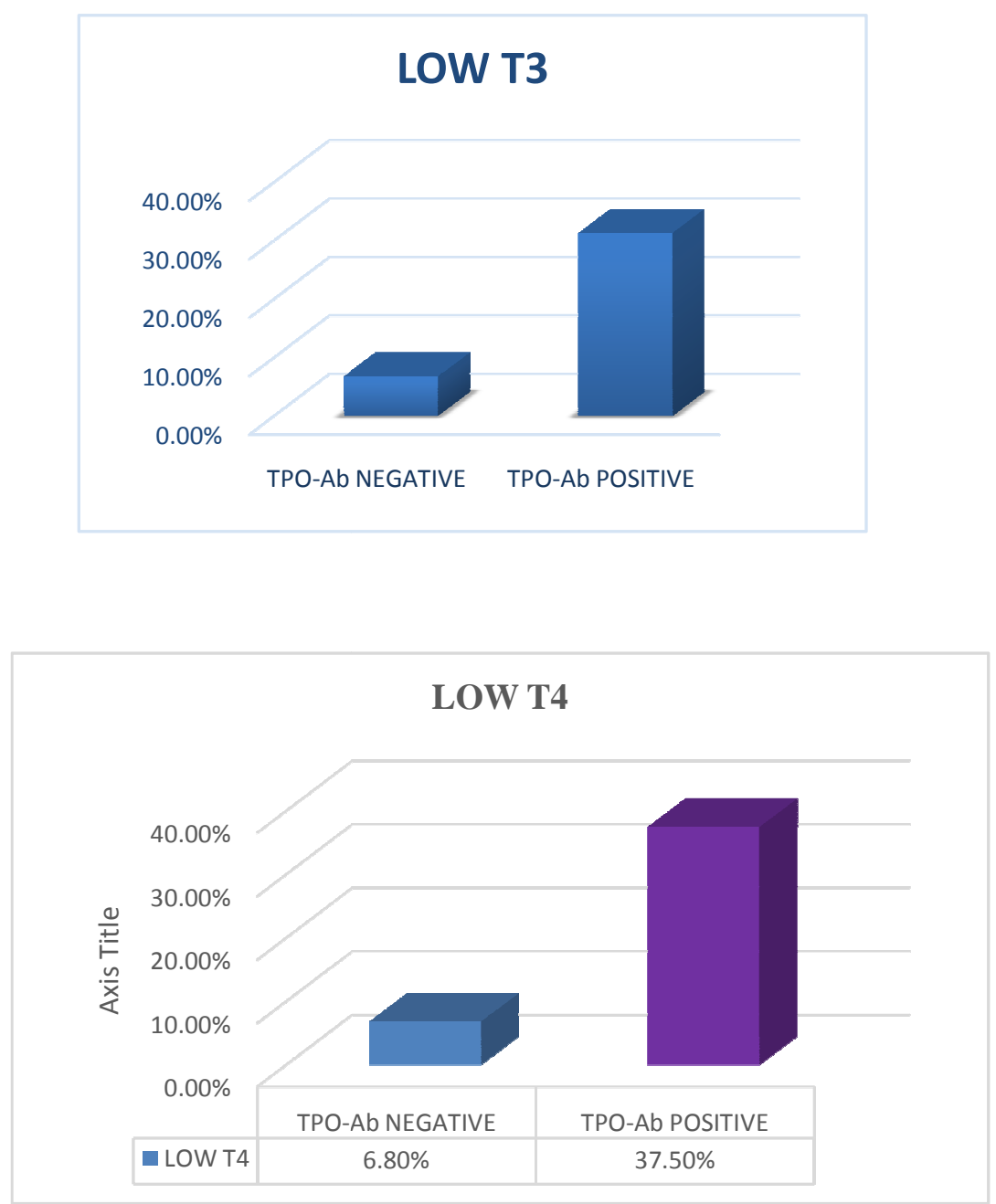


\section{Chart-4}

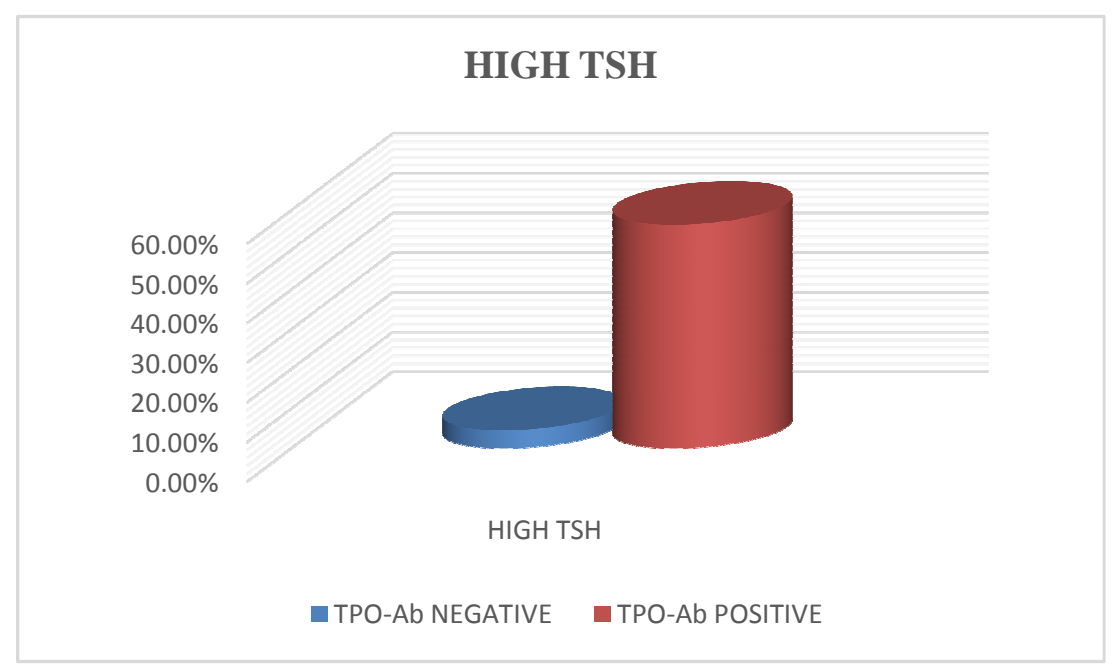

\begin{tabular}{|ll|r|r|r|r|r|r|}
\multicolumn{7}{|c|}{ Correlations } \\
\hline TPO & & TPO & AGE & DURATION & BLD_SUG & BMI & ATHRO_IN \\
\cline { 2 - 8 } & Pearson Correlation & 1 & .172 & .029 & -.076 & $.598^{* *}$ & .179 \\
& Sig. (2-tailed) &. & .189 & .823 & .566 & .000 & .172 \\
& $\mathrm{~N}$ & 60 & 60 & 60 & 60 & 60 & 60 \\
\hline AGE & Pearson Correlation & .172 & 1 & $.354^{* *}$ & .167 & $.407^{* *}$ & .046 \\
& Sig. (2-tailed) & .189 &. & .005 & .201 & .001 & .729 \\
& $\mathrm{~N}$ & 60 & 60 & 60 & 60 & 60 & 60 \\
\hline DURATION & Pearson Correlation & .029 & $.354^{* *}$ & 1 & .038 & .170 & -.210 \\
& Sig. (2-tailed) & .823 & .005 &. & .772 & .193 & .108 \\
& $\mathrm{~N}$ & 60 & 60 & 60 & 60 & 60 & 60 \\
\hline BLD_SUG & Pearson Correlation & -.076 & .167 & .038 & 1 & -.018 & -.105 \\
& Sig. (2-tailed) & .566 & .201 & .772 &. & .889 & .425 \\
& $\mathrm{~N}$ & 60 & 60 & 60 & 60 & 60 & 60 \\
\hline BMI & Pearson Correlation & $.598^{* *}$ & $.407^{* *}$ & .170 & -.018 & 1 & $.274^{*}$ \\
& Sig. (2-tailed) & .000 & .001 & .193 & .889 &. & .034 \\
& $\mathrm{~N}$ & 60 & 60 & 60 & 60 & 60 & 60 \\
\hline ATHRO_IN & Pearson Correlation & .179 & .046 & -.210 & -.105 & $.274^{*}$ & 1 \\
& Sig. (2-tailed) & .172 & .729 & .108 & .425 & .034 &. \\
& $\mathrm{~N}$ & 60 & 60 & 60 & 60 & 60 & 60 \\
\hline
\end{tabular}

**. Correlation is significant at the 0.01 level (2-tailed).

*. Correlation is significant at the 0.05 level (2-tailed). 
Table-4

\section{Correlations}

\begin{tabular}{|ll|r|r|r|r|r|}
\hline & & \multicolumn{1}{|c|}{ TPO } & \multicolumn{1}{c|}{ TGL } & \multicolumn{1}{c|}{ HDL } & \multicolumn{1}{c|}{ LDL } & \multicolumn{1}{c|}{ VLDL } \\
\hline TPO & Pearson Correlation & 1 & .032 & .043 & .057 & -.041 \\
& Sig. (2-tailed) &. & .810 & .743 & .668 & .756 \\
& $\mathrm{~N}$ & 60 & 60 & 60 & 60 & 60 \\
\hline TGL & Pearson Correlation & .032 & 1 & .115 & .030 & $.573^{\star}$ \\
& Sig. (2-tailed) & .810 &. & .380 & .821 & .000 \\
& $\mathrm{~N}$ & 60 & 60 & 60 & 60 & 60 \\
\hline HDL & Pearson Correlation & .043 & .115 & 1 & $.717^{\star *}$ & .053 \\
& Sig. (2-tailed) & .743 & .380 &. & .000 & .688 \\
& $\mathrm{~N}$ & 60 & 60 & 60 & 60 & 60 \\
\hline LDL & Pearson Correlation & .057 & .030 & $.717^{* *}$ & 1 & -.159 \\
& Sig. (2-tailed) & .668 & .821 & .000 &. & .224 \\
& $\mathrm{~N}$ & 60 & 60 & 60 & 60 & 60 \\
\hline VLDL & Pearson Correlation & -.041 & $.573^{\star *}$ & .053 & -.159 & 1 \\
& Sig. (2-tailed) & .756 & .000 & .688 & .224 &. \\
& $\mathrm{~N}$ & 60 & 60 & 60 & 60 & 60 \\
\hline
\end{tabular}

${ }^{* *}$. Correlation is significant at the 0.01 level (2-tailed).

Table-5

Correlations

\begin{tabular}{|c|c|c|c|c|c|c|}
\hline & & TPO & T3 & $\mathrm{T} 4$ & TSH & CHOLESTR \\
\hline \multirow[t]{3}{*}{ TPO } & Pearson Correlation & 1 & $-.296^{*}$ & $-.403^{\star *}$ & $.580^{\star \star}$ & .043 \\
\hline & Sig. (2-tailed) & & .022 & .001 & .000 & .746 \\
\hline & $\mathrm{N}$ & 60 & 60 & 60 & 60 & 60 \\
\hline \multirow[t]{3}{*}{ T3 } & Pearson Correlation & $-.296^{*}$ & 1 & $.457^{* *}$ & $-.585^{\star \star}$ & $-.257^{*}$ \\
\hline & Sig. (2-tailed) & .022 & . & .000 & .000 & .048 \\
\hline & $\mathrm{N}$ & 60 & 60 & 60 & 60 & 60 \\
\hline \multirow[t]{3}{*}{ T4 } & Pearson Correlation & $-.403^{* *}$ & $.457^{\star \star}$ & 1 & $-.768^{\star \star}$ & $-.480^{\star}$ \\
\hline & Sig. (2-tailed) & .001 & .000 & . & .000 & .000 \\
\hline & $\mathrm{N}$ & 60 & 60 & 60 & 60 & 60 \\
\hline \multirow[t]{3}{*}{ TSH } & Pearson Correlation & $.580 * *$ & $-.585^{\star \star}$ & $-.768^{* *}$ & 1 & $.368^{*}$ \\
\hline & Sig. (2-tailed) & .000 & .000 & .000 & . & .004 \\
\hline & $\mathrm{N}$ & 60 & 60 & 60 & 60 & 60 \\
\hline \multirow[t]{3}{*}{ CHOLESTR } & Pearson Correlation & .043 & $-.257^{*}$ & $-.480^{* *}$ & $.368^{\star *}$ & 1 \\
\hline & Sig. (2-tailed) & .746 & .048 & .000 & .004 & . \\
\hline & $\mathrm{N}$ & 60 & 60 & 60 & 60 & 60 \\
\hline
\end{tabular}

* . Correlation is significant at the 0.05 level (2-tailed).

**. Correlation is significant at the 0.01 level (2-tailed). 